\title{
Front Matter: Volume 7784
}

, "Front Matter: Volume 7784," Proc. SPIE 7784, Tenth International Conference on Solid State Lighting, 778401 (1 September 2010); doi: $10.1117 / 12.878987$

SPIE Event: SPIE Optical Engineering + Applications, 2010, San Diego, California, SPIE. United States 


\title{
PROCEEDINGS OF SPIE
}

\section{Tenth International Conference on Solid State Lighting}

\author{
lan Ferguson \\ Matthew H. Kane \\ Nadarajah Narendran \\ Tsunemasa Taguchi \\ Editors
}

2-4 August 2010

San Diego, California, United States

Sponsored and Published by

SPIE 
The papers included in this volume were part of the technical conference cited on the cover and title page. Papers were selected and subject to review by the editors and conference program committee. Some conference presentations may not be available for publication. The papers published in these proceedings reflect the work and thoughts of the authors and are published herein as submitted. The publisher is not responsible for the validity of the information or for any outcomes resulting from reliance thereon.

Please use the following format to cite material from this book:

Author(s), "Title of Paper," in Tenth International Conference on Solid State Lighting, edited by lan Ferguson, Matthew H. Kane, Nadarajah Narendran, Tsunemasa Taguchi, Proceedings of SPIE Vol. 7784 (SPIE, Bellingham, WA, 2010) Article CID Number.

ISSN 0277-786X

ISBN 9780819482808

Published by

SPIE

P.O. Box 10, Bellingham, Washington 98227-0010 USA

Telephone +1 3606763290 (Pacific Time) · Fax +1 3606471445

SPIE.org

Copyright (C) 2010, Society of Photo-Optical Instrumentation Engineers

Copying of material in this book for internal or personal use, or for the internal or personal use of specific clients, beyond the fair use provisions granted by the U.S. Copyright Law is authorized by SPIE subject to payment of copying fees. The Transactional Reporting Service base fee for this volume is $\$ 18.00$ per article (or portion thereof), which should be paid directly to the Copyright Clearance Center (CCC), 222 Rosewood Drive, Danvers, MA 01923. Payment may also be made electronically through CCC Online at copyright.com. Other copying for republication, resale, advertising or promotion, or any form of systematic or multiple reproduction of any material in this book is prohibited except with permission in writing from the publisher. The CCC fee code is 0277-786X/10/\$18.00.

Printed in the United States of America.

Publication of record for individual papers is online in the SPIE Digital Library.

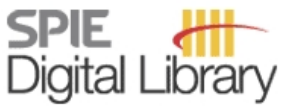

SPIEDigitalLibrary.org

Paper Numbering: Proceedings of SPIE follow an e-First publication model, with papers published first online and then in print and on CD-ROM. Papers are published as they are submitted and meet publication criteria. A unique, consistent, permanent citation identifier (CID) number is assigned to each article at the time of the first publication. Utilization of CIDs allows articles to be fully citable as soon they are published online, and connects the same identifier to all online, print, and electronic versions of the publication. SPIE uses a six-digit CID article numbering system in which:

- The first four digits correspond to the SPIE volume number.

- The last two digits indicate publication order within the volume using a Base 36 numbering system employing both numerals and letters. These two-number sets start with 00, 01, 02, 03, 04, $05,06,07,08,09,0 A, 0 B \ldots 0 Z$, followed by 10-1Z, 20-2Z, etc.

The CID number appears on each page of the manuscript. The complete citation is used on the first page, and an abbreviated version on subsequent pages. Numbers in the index correspond to the last two digits of the six-digit CID number. 


\section{Contents}

vii Conference Committee

\section{ALTERNATIVE DESIGN I}

778407 High-efficiency photonic quasi-crystal light emitting diodes incorporating buried photonic crystal structures [7784-06]

M. D. B. Charlton, Univ. of Southampton (United Kingdom); P. A. Shields, D. W. E. Allsop,

W. N. Wang, Univ. of Bath (United Kingdom)

778408 Energy efficient control of polychromatic solid state lighting using a sensor network [7784-07]

M. Aldrich, Massachusetts Institute of Technology (United States); N. Zhao, RWTH Aachen

Univ. (Germany); J. Paradiso, Massachusetts Institute of Technology (United States)

$778409 \quad$ Estimating the junction temperature of AC LEDs [7784-08]

Y. Liu, A. Jayawardena, T. R. Klein, N. Narendran, Rensselaer Polytechnic Institute (United States)

\section{SOLID STATE LIGHTING AND OLEDS PLENARY SESSION}

7784 OA What would Edison do with solid state lighting? (Plenary Paper) [7784-301]

I. T. Ferguson, The Univ. of North Carolina at Charlotte (United States); A. Melton, Georgia Institute of Technology (United States); T. XU, Georgia Institute of Technology (United States) and Huazhong Univ. of Science and Technology (China); M. Jamil, W. Fenwick, Georgia Institute of Technology (United States)

\section{OLEDS AND SSL: JOINT SESSION WITH CONFERENCE 7776}

7784 OB Nano-patterning for LED light extraction and for nanorod LED array formation (Invited Paper) [7784-09]

L. Y. Chen, Y. Y. Huang, C. S. Cheng, J. J. Huang, National Taiwan Univ. (Taiwan)

\section{GROWTH II}

7784 OD A comprehensive study on the parameters effecting color conversion in phosphor converted white light emitting diodes (Invited Paper) [7784-11]

C. Sommer, F. P. Wenzl, F. Reil, J. R. Krenn, JOANNEUM RESEARCH Forschungsgesellschaft $\mathrm{mbH}$ (Austria); P. Pachler, S. Tasch, LEDON Lighting Jennersdorf GmbH (Austria); P. Hartmann, JOANNEUM RESEARCH Forschungsgesellschaft mbH (Austria) 
$7784 \mathrm{OE} \quad$ Free-standing $\mathrm{GaN}$-based LEDs with $\mathrm{ALD}-\mathrm{Al}_{2} \mathrm{O}_{3} / \mathrm{Si}$ substrate removed by wet etching [7784-12]

M. Jamil, Georgia Institute of Technology (United States); T. Xu, Georgia Institute of Technology (United States) and Huazhong Univ. of Science and Technology (China); A. Melton, B. Jampana, T. Zaidi, Georgia Institute of Technology (United States); S. Liu, Huazhong Univ. of Science and Technology (China); I. Ferguson, Georgia Institute of Technology (United States) and The Univ. of North Carolina at Charlotte (United States)

7784 OF Reactor pressure: growth temperature relation for InN epilayers grown by high-pressure CVD [7784-13]

M. Buegler, Georgia State Univ. (United States) and Technische Univ. Berlin (Germany); S. Gamage, R. Atalay, J. Wang, I. Senevirathna, Georgia State Univ. (United States); R. Kirste, Technische Univ. Berlin (Germany); T. Xu, M. Jamil, Georgia Institute of Technology (United States); I. Ferguson, The Univ. of North Carolina at Charlotte (United States); J. Tweedie, R. Collazo, North Carolina State Univ. (United States); A. Hoffmann, Technische Univ. Berlin (Germany); Z. Sitar, North Carolina State Univ. (United States); N. Dietz, Georgia State Univ. (United States)

7784 OG Broad area side emission LED for high power application [7784-14] J. H. Teng, N. S. S. Ang, S. J. Chua, W. Liu, A*STAR Institute of Materials Research and Engineering (Singapore)

$7784 \mathrm{OH} \quad$ Optical, structural properties and experimental procedures of GaGdN grown by metalorganic chemical vapor deposition [7784-15]

I.-H. Hung, Y.-H. Lai, Z. C. Feng, National Taiwan Univ. (Taiwan); S. Gupta, T. Zaidi, Georgia Institute of Technology (United States); I. Ferguson, The Univ. of North Carolina at Charlotte (United States); W. Lu, Fisk Univ. (United States)

\section{ALTERNATIVE DESIGN II}

7784 OJ Physical constraints on the performance of LED lamp replacements for A-19, PAR-38 and MR-16 configurations [7784-17] J. W. Curran, LED Transformations, LLC (United States)

7784 OK Solid state lighting in buildings: status and future [7784-18] S. Paolini, Lunera Lighting Inc. (United States)

$7784 \mathrm{OL} \quad$ Application efficacy for comparing energy demand in lighting applications [7784-19] N. Narendran, J. P. Freyssinier, J. Taylor, T. Dong, R. Capó, Rensselaer Polytechnic Institute (United States)

7784 OM Advanced dimming strategy for solid state luminaires [7784-20]

S. Bęczkowski, Aalborg Univ. (Denmark)

\section{PHOSPHORS}

7784 OP Post-synthesis annealing effects on $\mathrm{SrGa}_{2} \mathrm{Se}_{4}: \mathrm{Eu}^{2+}$ phosphors with peak emission wavelength in the green gap [7784-23]

Y. Zhu, P. Dutta, N. Narendran, Rensselaer Polytechnic Institute (United States) 
7784 OR Philips' 2nd generation Novallure LED candle lamp [7784-49]

Y. Li, Z. Pei, C. Yuan, T. Jiang, Z. Lu, Y. Wang, X. Duan, Y. Xiong, H. Zhong, Y. Liu, Philips Lighting (China)

7784 OT Thermal transient characteristics of flip chip high power light emitting diodes [7784-27] C.-P. Wang, T.-T. Chen, S.-C. Yang, H.-K. Fu, A.-T. Lee, P.-T. Chou, C.-J. Sun, C.-L. Chen, M.-T. Chu, Industrial Technology Research Institute (Taiwan)

7784 OU LED luminaire longevity strategy models comparison [7784-28]

H. Lemieux, S. Thibault, Univ. Laval (Canada); A. A. Martel, Dellux Technologies (Canada)

7784 OV A two-metric proposal to specify the color-rendering properties of light sources for retail lighting [7784-29]

J. P. Freyssinier, M. Rea, Rensselaer Polytechnic Institute (United States)

\section{GROWTH III}

7784 OW Fully phosphor-converted LEDs with Lumiramic phosphor technology (Invited Paper) [7784-30]

H. Bechtel, P. J. Schmidt, A. Tücks, M. Heidemann, Philips Research Europe (Germany);

D. Chamberlin, R. Müller-Mach, G. O. Müller, O. Shchekin, Philips Lumileds Lighting Co. (United States)

$77840 Z$ High brightness GaN light emitting diodes with different barrier widths in quantum wells for general lighting application [7784-33]

I.-H. Hung, Y.-H. Lai, Z. C. Feng, National Taiwan Univ. (Taiwan); G. Li, Rainbow Optoelectronics Material Shanghai Co., Ltd. (China); I. Ferguson, The Univ. of North Carolina at Charlotte (United States); W. Lu, Fisk Univ. (United States)

778410 The effects of titania diffuser on angular color homogeneity in the phosphor conformal coated white LEDs [7784-34]

K.-C. Lee, S.-M. Kim, Korea Photonics Technology Institute (Korea, Republic of); J.-H. Moon, Chonnam National Univ. (Korea, Republic of)

\section{APPLICATION II}

778411 Concepts for future solid state lighting solutions (Invited Paper) [7784-35]

N. von Malm, R. Wirth, S. Illek, U. Steegmüller, OSRAM Opto Semiconductors GmbH (Germany)

778414 Emerging standard for thermal testing of power LEDs and its possible implementation [7784-38]

A. Poppe, Mentor Graphics Corp. (Hungary) and Budapest Univ. of Technology and Economics (Hungary); G. Farkas, G. Molnár, B. Katona, Mentor Graphics Corp. (Hungary); T. Temesvölgyi, Mentor Graphics Corp. (Hungary) and Budapest Univ. of Technology and Economics (Hungary); J.-W. He, Mentor Graphics Corp. (United States) 
778415 Optical, structural, and nuclear scientific studies of AIGaN with high Al composition [7784-39]

T. Y. Lin, Y. L. Chung, National Taiwan Univ. (Taiwan); L. Li, S. Yao, Peking Univ. (China); Y. C. Lee, Tung Nan Institute of Technology (Taiwan); Z. C. Feng, National Taiwan Univ. (Taiwan); I. T. Ferguson, The Univ. of North Carolina at Charlotte (United States); W. Lu, Fisk Univ. (United States)

778416 Rutherford backscattering and optical studies for $\mathrm{ZnO}$ thin films on sapphire substrates grown by metalorganic chemical vapor deposition [7784-40]

Y. L. Chung, National Taiwan Univ. (Taiwan); L. Li, S. Yao, Peking Univ. (China); Z. C. Feng, National Taiwan Univ. (Taiwan); W. E. Fenwick, T. Zaidi, Georgia Institute of Technology (United States); I. T. Ferguson, The Univ. of North Carolina at Charlotte (United States); W. Lu, Fisk Univ. (United States)

778417 A nanorods AIN layer prepared by sputtering at oblique-angle and application as a buffer layer in a GaN-based light emitting diodes [7784-41]

L.-C. Chen, C.-H. Tien, S.-Y. Chien, W.-C. Liao, C.-C. Huang, C.-S. Mu, C.-C. Chen, Y.-Y. Hsu, National Taipei Univ. of Technology (Taiwan)

778418 Effects of color temperature and luminous efficiency for RGB LEDs mixing with tuning voltage [7784-42]

H.-C. Chen, G.-Y. Wu, J.-Y. Lin, National Yunlin Univ. of Science and Technology (Taiwan)

778419 Assessment of ESD robustness in high power light emitting diodes [7784-43]

S.-C. Yang, P. Lin, National Chiao Tung Univ. (Taiwan); H.-K. Fu, C.-P. Wang, T.-T. Chen, A.-T. Lee, S.-B. Huang, P.-T. Chou, Industrial Technology Research Institute (Taiwan); W. Wang, F.-J. Kao, National Sun Yat-Sen Univ. (Taiwan)

7784 1A Effects of radiation characteristic and luminous efficiency for LED lampshade with cylindrical lens [7784-44]

H.-C. Chen, J.-Y. Lin, C.-H. Yang, Y.-X. Lu, National Yunlin Univ. of Science and Technology (Taiwan)

$77841 \mathrm{E} \quad$ Luminescence studies of $\mathrm{BaGd}_{2} \mathrm{O}_{4}: \mathrm{Tb}^{+3}$ phosphor [7784-48]

A. R. Bojja, S. Mudavat, S. Karnati, V. Muga, Osmania Univ. (India); B. M. Cheng, National Synchrotron Radiation Research Ctr. (Taiwan)

Author Index 


\title{
Conference Committee
}

\author{
Program Track Chair \\ Ian Ferguson, The University of North Carolina at Charlotte (United \\ States) \\ Conference Chairs \\ Ian Ferguson, The University of North Carolina at Charlotte (United \\ States) \\ Matthew H. Kane, University of Oklahoma (United States) \\ Nadarajah Narendran, Rensselaer Polytechnic Institute (United States) \\ Tsunemasa Taguchi, Yamaguchi University (Japan)
}

Program Committee

Srinath K. Aanegola, GE Lumination LLC (United States)

Andrew A. Allerman, Sandia National Laboratories (United States)

Ian E. Ashdown, byHeart Consultants Ltd. (Canada)

Lianghui Chen, Institute of Semiconductors (China)

John W. Curran, LED Transformations, LLC (United States)

Nikolaus Dietz, Georgia State University (United States)

Samuel Graham, Georgia Institute of Technology (United States)

Volker K. Härle, OSRAM Opto Semiconductors GmbH (Germany)

Christoph Hoelen, Philips Lighting B.V. (Netherlands)

Jianzhong Jiao, OSRAM Opto Semiconductors Inc. (United States)

Asif M. Khan, University of South Carolina (United States)

Michael R. Krames, Soraa, Inc. (United States)

Yung-Sheng Liu, National Tsing Hua University (Taiwan)

Eun-Hyun Park, SemiconLight Company, Ltd. (Korea, Republic of)

Seong-Ju Park, Gwangju Institute of Science and Technology (Korea,

Republic of)

Jeff Quinlan, Acuity Brands Lighting, Inc. (United States)

Robert V. Steele, Strategies Unlimited (United States)

Christian Wetzel, Rensselaer Polytechnic Institute (United States)

Chih-Chung Yang, National Taiwan University (Taiwan)

Session Chairs

1 Growth I

Christian Wetzel, Rensselaer Polytechnic Institute (United States)

2 Alternative Design I

John W. Curran, LED Transformations, LLC (United States) 
3 Solid State Lighting and OLEDs Plenary Session

Jianzhong Jiao, OSRAM Opto Semiconductors Inc. (United States)

Franky So, University of Florida (United States)

$4 \quad$ OLEDS and SSL: Joint Session with Conference 7776

Yuan-Sheng Tyan, Tyan Consulting (United States)

Matthew H. Kane, The University of Oklahoma (United States)

5 Growth II

Seong-Ju Park, Gwangju Institute of Science and Technology (Korea, Republic of)

6 Alternative Design II

Nikolaus Dietz, Georgia State University (United States)

$7 \quad$ Phosphors

Jianzhong Jiao, OSRAM Opto Semiconductors Inc. (United States)

8 Application I

Edward D. Petrow, Lincoln Technical Services, Inc. (United States)

9 Growth III

Jian-Jang Huang, National Taiwan University (Taiwan)

10 Application II

Matthew H. Kane, The University of Oklahoma (United States) 